\title{
Impacto de la deforestación en el desarrollo local sostenible de los hogares: caso de Nicaragua, 1998-2005
}

\section{Carlos Alberto Zúniga González*}

Recibido: noviembre de 2010 / Aceptado: enero de 2011

El artículo analiza la problemática de la deforestación y su impacto en el desarrollo local sostenible (DLS) durante el período 1998-2005. Una función de frontera estocástica es definida para variables específicas de panel de datos. Los efectos de ineficiencia técnica son asumidos para ser independientemente distribuidos como truncaciones de distribución normal con varianza constante pero con promedios, los cuales son una función lineal de variables observables. Se seleccionó un panel de datos de las bases de datos de las EMNV 98-05 (INIDE, 1998; 2005) eligiendo variables ambientales y sociales.

Los resultados evidencian que la problemática de la deforestación es explicada por los gastos sociales y la pobreza. El promedio de la eficiencia técnica fue del $22 \%$ y la pobreza alcanzó niveles significantes para explicar la ineficiencia técnica en el modelo de frontera estocástica. La eficiencia técnica por año fue de 27\%, 35\% y 5\% durante los años 1998, 2001 y 2005 respectivamente. Bajo estas consideraciones sugerimos en la política ambiental un proceso educativo y de especialización de los productores(as) de cara a mitigar los efectos de esta problemática.

Palabras clave: LSMS Survey / MECOVI / eficiencia técnica / frontera estocástica / Desarrollo Local Sostenible 


\section{Introducción}

En Nicaragua se han elaborado muchos estudios para identificar los problemas ambientales relacionados con la agricultura y la forestería. Entre estos problemas podemos mencionar a) la calidad de agua y acceso al consumo humano, irrigación y energía, b) la deforestación, c) la contaminación ambiental y el control de toxinas, d) el deterioro y erosión de suelos, y e) la pérdida de biodiversidad/pérdida de potencial en servicios ambientales. En estos estudios se ha identificado la deforestación como el problema de más alta frecuencia y en segundo orden, la escasez de fuentes de agua (Urbina, 2005).

A partir de 2007, el nuevo gobierno aborda esta problemática mediante la declaración de "La Madre Tierra", donde el nuevo modelo de participación ciudadana tiene incidencia a través de los programas que desarrollan las instituciones de los gabinetes de producción social y ambiental (Zúniga, 2010a). Sin embargo, la problemática de la deforestación presenta entre sus principales causas la limitada capacidad administrativa para el manejo de los bosques (infraestructura, control, estándares); la deforestación como resultado del avance de la frontera agrícola; la falta de capacidad para la administración y ejecución de planes de restauración; el fracaso para valorar los recursos naturales como base del desarrollo; la insuficiente información, investigación y monitoreo de los bosques nacionales para su administración eficiente; los conflictos de interés e incertidumbre en los derechos de propiedad de tierras; la forestería industrial con tecnología pobre y obsoleta; la alta frecuencia en quemas; y las plagas sin control en bosques de pinos de altura, implicando un deterioro del paisaje natural (Gómez Sal, 2004). Estas causas producen un efecto negativo en los subsistemas de producción, haciendo insostenible las actividades productivas, incrementando la extracción ilegal de madera, la degradación de las cuencas y fuentes de agua, los niveles de pobreza, las pérdidas en la utilización y transformación de la madera, las pérdidas en la calidad genética de los recursos del bosque, la reducción del potencial hidroeléctrico en la cuencas deforestadas, entre otras (Urbina, 2005).

Este estudio pretende contribuir a la política ambiental valorando el impacto de la deforestación como una problemática ambiental en el desarrollo local sostenible. El estudio considera evaluar el impacto de la tala de árboles como actividad económica del subsistema de producción forestal para el desarrollo local sostenible y analizar sus posibles causas.

En la organización de nuestro trabajo se hace una revisión de la teoría del modelo de desarrollo local sostenible relacionado con la problemática de la deforestación como un medio de subsistencia y el modelo de efectos en la ineficiencia técnica del modelo de frontera estocástica. Luego se presenta una descripción de la aplicación empírica, donde se explican los datos y la metodología utilizada, Finalmente se presentan los resultados, las conclusiones y discusión con sus respectivas recomendaciones. 


\section{Revisión de la literatura}

La revisión de la literatura se hizo en dos direcciones. Primeramente una revisión de la especificación del modelo de la función de producción de frontera estocástica, donde se considera la especificación del modelo como una función de producción de frontera estocástica que se aplica desde los años setenta (Aigner, Lovell \& Schmidt, 1977; Meeusen \& van den Broeck, 1977). En un segundo momento se revisó el tema del modelo de desarrollo local sostenible (Gómez Sal, 2004).

En la primera parte de la revisión se destaca la contribución científica a la metodología de la función de frontera estocástica para evaluar o medir la eficiencia técnica. La original especificación del modelo razonó una función de producción detallada para datos de cruce seccional, el cual tuvo un término error de dos componentes, uno para calcular los efectos aleatorios y el otros para calcular la ineficiencia técnica. Este modelo, denominado Battese y Coelli (1995), fue utilizado como modelo de frontera de ineficiencia para panel de datos y fue expresado como ec(1):

$$
Y_{i t}=\exp \left(\mathrm{X}_{\mathrm{it}} \beta+\left(V_{i t}-\mathrm{U}_{\mathrm{it}}\right)\right)
$$

$, i=1 \ldots . ., N, t=1 \ldots \ldots \ldots \ldots T$, donde $Y_{i p}, X_{i p}, y \beta$ son calculados en la primera fase del Programa Frontier 4.1 y donde:

$Y_{i t}$, es la producción o el logaritmo natural de la producción de la i-ésimo finca estudiada $(\mathrm{t}=1,2 \ldots \ldots \mathrm{N})$;

$X_{i t}$, es un vector (1xk) de transformaciones de las cantidades de input (o sus valores) de la i-ésimo finca estudiada en la t-ésima observación;

$\beta$, es un vector $(\mathrm{kx} 1)$ de parámetros desconocidos a estimar;

Las variables aleatorias Vit son asumidas para ser determinada iid (independientes e idénticamente distribuidos) del tipo, $\mathrm{N}\left(0, \sigma_{v}^{2}\right)$ e independiente de $U_{i t}$ las cuales son variables aleatorias no negativas que son asumidas para contabilizar la ineficiencias técnicas en la producción, además son asumidas para obtener una distribución truncadas independientes en cero de la distribución $\left|N\left(m_{i t}, \sigma_{\mathrm{u}}^{2}\right)\right|$, donde

$$
\text { mit }=Z_{i t} \delta
$$

,donde zeta $\left(Z_{i t}\right)$ es un vector de 1 x p variables las cuales pueden influir en la eficiencia técnica de la finca y delta $(\delta)$ es un vector de p X 1 parámetros a ser estimados.

La ec(1) específica la función de producción de frontera estocástica en términos de los valores originales de la producción original. Sin embargo, para los efectos de la ineficiencia técnica $U_{i t}$, es asumida para ser una función de un conjunto de variables explicativas, $\mathrm{Z}_{\mathrm{it}}$, $\mathrm{y}$ un vector de coeficientes desconocidos, $\delta$. 
Las variables explicativas en el modelo de ineficiencia técnica pueden incluir algunas variables input en la frontera estocástica, suministrado con efectos de ineficiencia que son estocásticos. Si la primera variable $z$ tiene un valor de 1 y todos los otros coeficientes son 0 , entonces este caso representa el modelo especificado por Stevenson (1980) y Battese y Coelli $(1988,1992)$. Si todos los elementos del vector $\delta$ son igual a 0 , entonces los efectos de la ineficiencia técnica no están relacionados con las variables z y tienen distribución semi-normal originalmente especificada por Aigner, Lovell y Schmidt (1977). Si las interacciones entre las específicas variables de fincas y las variables input son incluidas como variables $z$, entonces es obtenida una frontera estocástica no neutral (Huang \& Liu, 1994).

Los efectos de la ineficiencia técnica, $\mathrm{U}_{\mathrm{it}}$, en el modelo de frontera estocástica (ec. 1) puede ser especificado como ec (3):

$$
U_{i t}=Z_{i t} \delta+W_{i t}
$$

, donde la variable aleatoria, $W_{i t}$, es definida como distribución normal trucada en 0 por medio de la varianza, $\sigma^{2}$, de tal manera que tal punto de truncación es $-Z_{\text {it }} \delta$, por ejemplo $\mathrm{W}_{\mathrm{it}} \geq-\mathrm{Z}_{\mathrm{it}} \delta$. Estos supuestos son consistentes con $U_{i t}$ siendo una truncación no negativa de la distribución $N\left(z_{i t} \delta, \sigma^{2}\right)$.

El método de máxima verosimilitud (MLE) es propuesto para estimaciones simultáneas de los parámetros para los modelos de frontera estocástica y efectos de ineficiencia técnica. La función de verosimilitud (LLF) y su derivada parcial con respecto a los parámetros del modelo presentado en Battesse y Coelli (1992). La función de verosimilitud es expresada en términos de los parámetros de varianza, $\sigma^{2}$ $=\sigma_{v}^{2}+\sigma_{\mathrm{u}}^{2}$ y gamma $\gamma=\sigma_{\mathrm{u}}^{2} / \sigma_{v}^{2}+\sigma_{\mathrm{u}}^{2}$.

La ineficiencia técnica de producción para la i-ésima finca en la t-ésima observación es definida como ec (4):

$$
\mathrm{TEit}=\exp (\mathrm{Uit})=\exp (-\mathrm{Zit} \delta-\mathrm{Wit})
$$

En las últimas dos décadas encontramos que esta especificación original ha sido usada en un vasto número de aplicaciones. La especificación también ha sido modificada y extendida de varias maneras. Estas extensiones incluyen asumir una especificación de distribución más general para $U_{i t}$, tal como una distribución normal truncada o una distribución gama $(\gamma)$, como se explicó anteriormente, además, la consideración de panel de datos y la variación del tiempo de la eficiencia técnica; la extensión de la metodología a función de costos y también la estimación de sistemas de ecuaciones (Forsund, Lovell \& Schmidt, 1980; Schmidt, 1986; Baurer, 1990; Greene, 1993).

Kumbhakar, Ghosh y McGuckin (1991) y Reifschneider and Stevenson (1991) propusieron un modelo en el cual los efectos de la ineficiencia $U_{\text {it }}$ son expresados como una función explícita de un vector de variables de fincas ${ }^{2}$ específicas y un error aleatorio. Battese y Coelli (1995) propusieron un modelo en el cual retoma la especificación de los autores anteriores, con la excepción que la eficiencia asignativa es impuesta, el primer orden remueve la condición de maximización de beneficios, y el panel de datos es permitido. Este modelo puede 
ser definido como (ec.1), reemplazando $\sigma_{v}^{2}$ y $\sigma_{u}^{2}$ con sigma cuadrado $\left(\sigma^{2} \equiv \sigma_{v}^{2}+\sigma_{u}^{2}\right)$ y gamma $\left(\gamma \equiv \sigma_{u}^{2} / \sigma_{v}^{2}+\sigma_{u}^{2}\right)$, (Battese \& Coelli, 1995).

Algunos errores en la interpretación suelen ocurrir al estudiar los resultados de este error compuesto. La variable $U_{\text {it }}$ puede ser especificado con las distribuciones Exponencial, Seminormal, Normal Truncada y Gamma. En el caso que $U_{i t}$ se especifique como Seminormal o Normal Truncada, la relevancia de la ineficiencia del sistema puede ser sobrevalorada debido a una mala interpretación de los parámetros $\lambda$ у $\gamma$ (Dios Palomares, 2002).

La segunda temática en la revisión de la literatura se hizo sobre el desarrollo local sostenible (Gómez Sal, 2004). Los trabajos científicos sobre este tema tienen como antecedente los años sesenta, donde se observa el nacimiento de una corriente del pensamiento entorno al modelo de desarrollo local sostenible. Se empiezan a relacionar las ideas de ecodesarrollo, desarrollo alternativo, nuevo desarrollo o meta desarrollo (Valcárcel-Resalt, 1992), introduciendo un nuevo enfoque de desarrollo: enfoque local.

Dentro del pensamiento internacional se destacan las aportaciones que desde la geografia se han hecho al desarrollo local y su implementación a los espacios rurales (Meilan Gil, 1971; Faure, 1979; Hoggart, 1987; Potter \& Unwng, 1989), de igual manera encontramos estudios sobre política de desarrollo local (Atienza Serna, 1992; B.I.R.F, 1975; Cordero Mestanza, 1990; Entrena Durán, 1992; FAO,1993). Especial interés tienen los diversos estudios sobre grupos sociales y la importancia de la mujer en el mundo rural (Zapata Martelo, 1994; Kayser, 1990; Chombart de Lauwe, 1988), al igual que la participación social donde se destaca el papel de los actores y agentes locales (Berger, 1992; Oakley et al., 1993). Otra aportación al desarrollo local fueron los estudios sobre el mercado de trabajo y creación de empleo en las zonas rurales (Boekena, 1989; Carbonell, 1992; Sambergs, 1979). La importancia de la cultura en la formación del mundo rural es también mencionada en la literatura del desarrollo local (Fortun, 1973; Chombart de Lauwe, 1988; Rodríguez Fraguas, 1993). Es meritorio mencionar los estudios referentes a las comunicaciones y a las nuevas tecnologías que contribuyeron y contribuyen al desarrollo rural (Kenneth Dyson, 1998; Ruíz Pérez, 1993). Con relación al turismo rural también destaca el aporte científico, aunque no numeroso, considerable por su valor (Crosby, 1993 y 1994; Bote Gómez, 1990). La preocupación por el medio ambiente, por la mejora del paisaje natural, ha sido un aporte del pensamiento internacional (Bifani, 1984; Whatmore, Marsder \& Lowe, 1993). Otro grupo de investigadores ha realizado estudios sobre la evaluación de modelos de desarrollo (Barke \& Newton, 1995; Gavira Álvarez, 1993).

La idea de un modelo multidimensional del desarrollo que reconoce el papel del medio ambiente es la retomada en este trabajo (Gómez Sal, 1998 y 2001). En él se consideran las variables ecológica, productiva, económica, social, cultural y ética, donde se analiza la contribución al desarrollo local sostenible desde una perspectiva ecológica (Gómez Sal, 2004). 


\section{Aplicación empírica}

Se utilizaron las bases de datos de las encuestas de mejoramiento de nivel de vida (INIDE, 1998-2005), de las cuales seleccionamos 20 casos por cada año, construyendo un panel de datos de 60 casos en total. El criterio de selección fue identificar en las tres bases de datos los mismos hogares, es decir que los hogares seleccionados de la base de 1998 son los mismos del 2001 y del 2005, además que estos hogares tuvieran fincas forestales y que las mismas cumplieran con las condiciones del modelo de función de frontera estocásticas. Tales criterios son: generar ingresos y haber definido un costo de producción (abonos orgánicos). Los casos que no cumplían con estos criterios no fueron seleccionados. Esta selección es parte del esfuerzo por mejorar la diseminación de las estadísticas de los hogares rurales y urbanos donde se presenta la oportunidad para integrar las estadísticas agropecuarias al análisis del desarrollo rural (Zuniga, 2010b; Carletto, 2010). Es importante señalar que la construcción del panel de datos nos permitió evaluar en el tiempo el comportamiento de la eficiencia técnica en cada finca estudiada al igual que el efecto de la ineficiencia técnica. El esfuerzo se centró en seleccionar las variables adecuadas y deflactarlas para incluir en el modelo de función frontera estocástica con un criterio ecológico que permitiera influir en el impacto del desarrollo local y sostenible de los hogares, que lograse medir el impacto de la tala de árboles como actividad económica y el efecto de la ineficiencia técnica que envolvió la pobreza y el consumo social como variables explicativas de la problemática (tala irracional). Para ello se procesó la información en el software SPSS y Frontier 4.1.

La metodología utilizada es la conocida como frontera estocástica de producción, que se basa en la función Coob Douglas (Battese \& Coelli, 1992 y 1995). Esta es una aplicación del modelo de Battese y Coelli (1995).

Se utilizó el programa de computación FRONTIER Versión 4.1 (Battese \& Coelli, 1988, 1992 y 1995) para obtener una estimación de máxima probabilidad (MLe) del panel de datos seleccionado en cada período de estudio; esta es planteada en la sección revisión de literatura. El modelo utilizado en base a la ec. 1, es el siguiente ec. 5:

$$
\ln \left(Q_{i}\right)=\beta_{0}+\beta_{1} \ln \left(J_{i}\right)+\beta_{2} \ln \left(S_{i}\right)+\left(V_{i}-U_{i}\right)
$$

, donde $\left(Q_{i}\right)$ representa el valor deflactado de la venta y autoconsumo por concepto de tala de árboles para cada una de las fincas estudiadas, durante el periodo 1998-2005.

$(J)$ Representa el valor deflactado de los jornales sin alimentación, con alimentación y trabajadores permanentes pagados en cada una de las fincas estudiadas, durante el periodo 1998-2005.

(S) representa la razón de cobertura del costo de la canasta básica como variable social del consumo o el gasto que realiza cada hogar en concepto de gastos de alimentos, vivienda, salud, educación, transporte, entre otros, correspondiente a cada año de estudio, durante 1998-2005. Se calculó dividiendo el ingreso total anual por hogar entre el costo anual de la canasta básica con respecto al año de estudio.

$\left(V_{i}-U_{i}\right)$, el componente del error compuesto $V i$ Representa variables 
aleatorias las cuales son asumidas para ser una distribución normal en $\mathrm{N}\left(0, \sigma_{v}^{2}\right)$ e independiente de $U_{i}$ quien representa variables aleatorias no negativa las cuales son asumidas para medir la ineficiencia técnica en la producción y es asumida para ser distribuida independiente como truncaciones en cero de la distribución normal $\mathrm{N}(\mathrm{mit}, \gamma)$ ec2. Estas medidas se interpretan como indicadores de la importancia relativa de cada variable en la composición del error compuesto, de tal manera que si gamma toma un valor próximo a 1 , se deduce que no hay efectos en el error debido a factores fuera del control de las fincas estudiadas (Dios: 2002).

$$
U_{i t}=\delta_{0}+\delta_{1}(E)+\delta_{2}(P)+W_{i t}
$$

Los efectos de la ineficiencia técnica son asumidos para ser definidos por la ec 6:

,donde $U_{i t}$, es el término error que mide el efecto de la ineficiencia técnica, explicada en la sección anterior.

$(E)$ Representa el costo deflactado de la utilización de abonos orgánicos en cada una de las fincas estudiadas, durante el periodo 1998-2005.

(P) representa una variable dicotómica: 0 si el hogar es pobre, 1 si el hogar no es pobre. Este nivel de pobreza es estimado en base a la línea de pobreza del año respectivo, durante 1998-2005.

$\left(W_{i t}\right)$ es la variable aleatoria explicada en la sección anterior.

La hipótesis a comprobar: Si el modelo de ineficiencia es estocástico, entones la eficiencia técnica puede ser explicada por el modelo de desarrollo local sostenible influenciada por la problemática de la tala de árboles.

\section{Resultados empíricos y análisis}

Esta sección describe los resultados de la estimación de los parámetros de frontera estocástica y los efectos de la eficiencia técnica en el modelo estocástico, así como la comprobación del modelo de ineficiencia para fincas forestales. Estos parámetros son basados en la función Cobb Douglas con frontera estocástica y el modelo de desarrollo local sostenible.

Los resultados fueron organizados en los cuadros 1-6. En el primer cuadro se hizo un resumen de las estadísticas descriptivas del panel de los datos por cada período estudiado. 
Cuadro 1. Estadísticas descriptivas del panel de datos 1998-2005

\begin{tabular}{|c|c|c|c|c|c|}
\hline Estadísticos & Output (Q) & Jornales (J) & Ecólogica (E) & Social (S) & Pobreza (P) \\
\hline Media & $1,099.25$ & $11,250.22$ & 91.00 & $35,096.95$ & 0.43 \\
\hline Desviación estándar & $2,313.50$ & $36,280.96$ & 330.31 & $27,571.59$ & 0.50 \\
\hline Varianza de la muestra & $5352,259.17$ & $1316308,157.39$ & $109,104.07$ & $760192,840.73$ & 0.25 \\
\hline Curtosis & 36.99 & 34.17 & 24.46 & 0.93 & -1.99 \\
\hline Coeficiente de asimetría & 5.61 & 5.44 & 4.66 & 1.09 & 0.28 \\
\hline Rango & $16,800.00$ & $252,504.00$ & $2,100.00$ & $125,526.67$ & 1.00 \\
\hline Mínimo & 0.00 & 0.00 & 0.00 & 0.00 & 0.00 \\
\hline Máximo & $16,800.00$ & $252,504.00$ & $2,100.00$ & $125,526.67$ & 1.00 \\
\hline Suma & $65,955.00$ & $675,013.00$ & $5,460.00$ & $2105,816.82$ & 26.00 \\
\hline Cuenta $(\mathrm{N})$ & 60.00 & 60.00 & 60.00 & 60.00 & 60.00 \\
\hline Mayor (1) & $16,800.00$ & $252,504.00$ & $2,100.00$ & $125,526.67$ & 1.00 \\
\hline Menor(1) & 0.00 & 0.00 & 0.00 & 0.00 & 0.00 \\
\hline Nivel de confianza(95.0\%) & 597.64 & $9,372.37$ & 85.33 & $7,122.50$ & 0.13 \\
\hline
\end{tabular}

En el cuadro 2 se presentan los parámetros de máxima verosimilitud de la producción forestal y los efectos de la ineficiencia técnica de acuerdo al modelo propuesto de desarrollo local sostenible.

El resultado del modelo especificado directamente es influenciado por el número de variables ambientales estudiadas (Battese \& Coelli, 1995). De la ecuación (3) obtuvimos:

\section{Frontera estocástica}

$\operatorname{Ln}(Q)=9.24+0.12 \operatorname{Ln}(J)+0.58 \operatorname{Ln}(S)(0.92)(0.07)$

Modelo de ineficiencia técnica

$U=-10.36-0.01(E)+6.62(P)(15.77)(0.02)$

\section{Parámetros de varianza}

$$
\sigma^{2}=44.12 \quad \gamma=0.98 \quad \text { (31.07) }
$$

La estimación de máxima verosimilitud (MLe) fue un procedimiento de maximización analítica de las variables utilizadas, implicando a cada forma de datos censurados o multicensurados. Fue posible usar varios cruces de técnicas estrechando celdas y estimar parámetros del modelo de contribución de la eficiencia técnica al desarrollo local sostenible (Battese \& Coelli, 1995).

Los coeficientes pueden ser interpretados como elasticidades. Los signos resultaron como se esperaba. El modelo de frontera estocástica puede interpretarse como una versión de la función de producción forestal Cobb Douglas aplicado a la actividad forestal. Es importante aclarar que los hogares seleccionados con fincas forestales están destinados a cultivos temporales, sin embargo los productores(as) realizaron tala de árboles, situación que evaluamos como impacto en la eficiencia técnica.

El coeficiente $\left(\beta_{0}\right)$ indica que es un valor imparcial de (9.24). Pudiese representar la contribución (elasticidad) al modelo (DLS) de eficiencia técnica 
cuando en el modelo se supone que no se utilizan jornales y no hay contribución al costo de la canasta básica, es decir, el sistema arbóreo se desarrolla de forma natural. Se puede interpretar como la contribución de C\$9.24 córdobas cuando no se invierte en este tipo de actividad económica. Este parámetro es significativo al nivel del $1 \%$ para el periodo del 98-2005, porque el valor de su estadístico t es mayor que 2.3936 en valor absoluto con 57 grados de libertad.

Cuadro 2. Parámetros de Máxima Verisimilitud estimados (MLE) de la producción forestal con frontera estocástica para el modelo de desarrollo local sostenible (MSLD), período 1998-2005

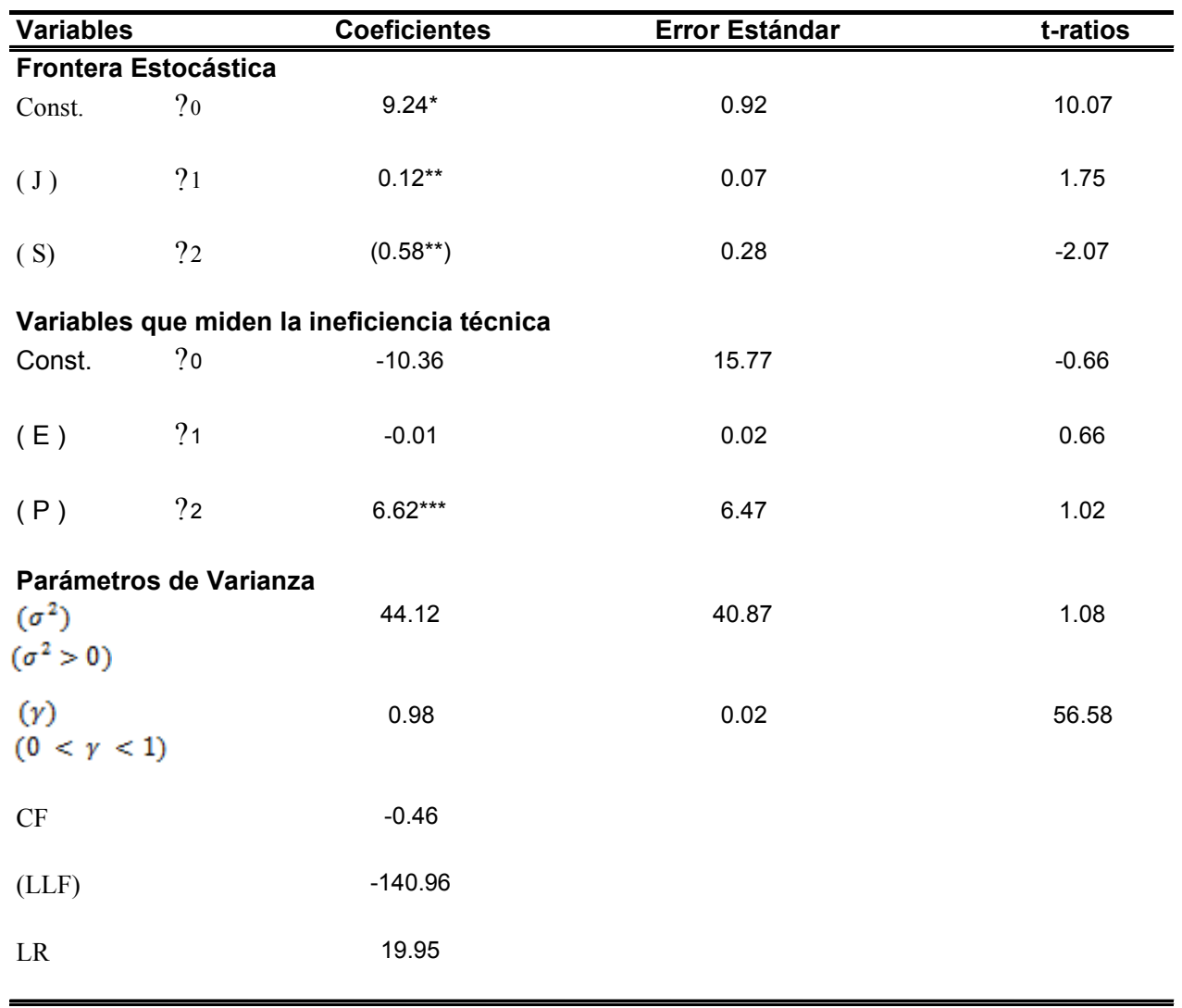

Jornales (J ), Ecológica ( E ), Social ( S ), Pobreza ( P )

Delta ?0, Delta ?1 Delta ?2

Sigma Cuadrado $\left(\sigma^{2}\right) \quad$, Gamma $(\gamma)$, Función del registro verosimilitud (LLF) Prueba Ratio likelihood (LR)

$\mathrm{CF}:$ Coeficiente de función $\quad * 1 \%$ nivel de significancia, $* * 5 \%$ nivel de significancia, $* * * 10 \%$ nivel de significancia

LLF y LR tiene un ajuste de la distribución Chi quadrado

El coeficiente $\left(\beta_{1}\right)$ indica que la contribución de los jornales con y sin alimentación como de trabajadores permanentes fue de $12 \%$; su elasticidad se interpreta expresando que por cada córdoba invertido en jornales para la tala se obtiene C $\$ 12$ córdobas. Este parámetro es significativo al nivel del 5\% para el periodo del 98 2005 porque el valor de su estadístico t es cercano a 1.6720 en valor absoluto con 57 grados de libertad. 
El coeficiente $\left(\beta_{2}\right)$ indica la contribución a reducir el costo de la canasta básica, su signo negativo se interpreta como una aporte inelástico, es decir que independientemente del aporte por la tala de árboles el hogar no logra cubrir el costo de la canasta básico, pues apenas alcanza el $58 \%$. Este parámetro es significativo al nivel del $5 \%$ para el periodo del 98-2005, porque el valor de su estadístico t es cercano a 1.6720 en valor absoluto con 57 grados de libertad.

El coeficiente de la función para el modelo de desarrollo local sostenible con frontera estocástica en promedio geométrico fue-0.46 revelando un tamaño suboptimizado a rendimientos de escala decreciente, es decir que los productores(as) de hogares con fincas forestales desarrollaron sus actividades en un tamaño no adecuado como para permitir la regeneración arbórea y por ende de ingresos, incidiendo en los niveles de ineficiencia, siendo una de las causas de la problemática de la tala de árboles. Ello se explica a que el destino de la fincas es para cultivos temporales más que para bosques.

Con respecto al modelo de efectos en la ineficiencia para el panel de datos de hogares con fincas forestales 1998-2005, los signos se expresan como se esperaba.

El coeficiente $\left(\delta_{0}\right)$ representa el nivel de ineficiencia del modelo propuesto sin considerar el efecto del ecológico ni la pobreza de los hogares con fincas forestales. Este coeficiente no es técnicamente significativo.

El coeficiente $\left(\delta_{1}\right)$ nos explica la ineficiencia del modelo con frontera estocástica por factores ecológicos. El signo negativo indica un impacto deficiente en la mano de obra utilizada, es decir la no inversión en abonos orgánicos reduce la eficiencia técnica de los jornales utilizados y por consiguiente no alcanza los suficientes ingresos para cubrir la canasta básica. Este parámetro no es técnicamente significativo.

El coeficiente $\left(\delta_{2}\right)$ es una variable dicotómica situación que dificulta su interpretación. Este coeficiente indica que la pobreza $(\mathrm{P})$ también es incidente para explicar los efectos de la ineficiencia en el MDL con frontera estocástica. La elasticidad es alta, es decir que el problema de la tala de árboles se debe fundamentalmente a los niveles de pobreza extrema, media y alta afectando el MDLS. Su coeficiente no es significativo, su valor es cercano al nivel de significancia del 10\%.

\section{Interpretación de los parámetros de variancia}

El valor del coeficiente de sigma cuadrado $\left(\sigma^{2}\right)$ es utilizado para examinar la distribución normal de la variable aleatoria de $\mathrm{Vi} \sim \mathrm{N}(0,7.57)$, este parámetro se interpreta como el primer componente del término error que explica los fenómenos ajenos a la voluntad de las unidades de producción forestal estudiadas y al igual que la distribución de gamma $(\gamma)$ es utilizado para medir el nivel de ineficiencia considerando la distribución truncada de $\mathrm{Ui} \sim \mathrm{N}\left(m_{i t}, 0.98\right)$. El valor de gamma $(\gamma)$ es considerado de 0.1 a 0.9 en incrementos de 0.1 ; el tamaño de este incremento puede ser alterado por la asignación que le indiquemos en el programa FRONTIER 4.1 (Battese \& Coelli, 1995). Por lo tanto, podemos indicar el nivel de ineficiencia en la especificación del modelo cuando tiene una variación para el período de estudio. El valor de 0.97 de gamma es cercano a uno, deduciéndose que los efectos de ineficiencia son probablemente de alta significancia en el análisis del valor output (ingresos generados por la tala de árboles) de los productores(as) forestales. Esto 
indica que los efectos de ineficiencia son probablemente de alta significancia en el estudio de la problemática de la tala de árboles.

El término error del modelo de efectos de ineficiencia es la variable aleatoria, $W_{i t}$; ésta es definida como la distribución normal que truncaría en 0 por medio de la varianza, $\sigma^{2}$, de tal manera que tal punto de truncación es $-Z_{i t} \delta$, por ejemplo $W_{i t}$ $\geq \mathrm{Z}_{\mathrm{it}} \delta$.

Generalizando la prueba de razón de probabilidad (ratio de likelihood) de la hipótesis nula, los efectos de ineficiencia están ausentes o tienen la más simple distribución (ver Cuadro 3). La primera hipótesis nula, que especifica que los efectos de la ineficiencia están ausentes del modelo, es fuertemente rechazada, es decir que las variables propuestas para explicar la problemática de tala son válidas. La segunda hipótesis nula, que especifica que los efectos de ineficiencia no son estocásticos, también fue fuertemente rechazada.

Cuadro 3. Prueba de hipótesis para los parámetros del modelo de frontera de ineficiencia para

\begin{tabular}{llccccc}
\hline $\begin{array}{l}\text { Hipótesis Nula } \\
\text { Ho }\end{array}$ & $\begin{array}{l}\text { Log } \\
\text { likelihood } \\
\text { Function }\end{array}$ & $\begin{array}{l}\text { Test Statistic } \\
\text { LR }(\boldsymbol{\lambda})\end{array}$ & Valor $\mathbf{0 . 9 5} \chi_{\text {.j.l) }}^{2}$ & Decisón & Alternativa \\
\hline $\mathrm{H} 0=\gamma=? 0=\ldots=2=0$ & -140.96 & $19.95^{*}$ & 9.4877 & (4) & Rechazar Ho & Efectos de ineficiencia \\
$\mathrm{H} 0=\gamma=0$ & -141.00 & $19.88^{*}$ & 9.4877 & $(4)$ & Rechazar Ho & Estócastica \\
$\mathrm{H} 0=? 1=? 2=0$ & -142.26 & $17.36^{*}$ & 9.4877 & (4) & Rechazar Ho & Función Lineal
\end{tabular}

*Significativo al nivel del $5 \%$. Con (g.l) igual al número de parametros asumidos para ser cero en la Ho $\lambda=-2 *\{\log [$ likelihood (Ho) $]-\log [$ likelihood (H1) $]\}$, tiene aproximandamente una distribucion Chi-cuadrada

La tercera hipótesis, considerada en el cuadro 3, especifica que los efectos de ineficiencia no son una función lineal de la variable ecológica (E) ni la pobreza (P) de las fincas en los años estudiados. Esta hipótesis nula es también rechazada en el nivel del 5\% de significancia. Esto indica que el modelo propuesto es válido para comprender la problemática de la tala de árboles, donde los jornales son significativos más que la contribución a cubrir el costo de la canasta básica. Los niveles de ineficiencia en la mano de obra en parte se explican por la necesidad de contribuir al impacto positivo ecológicamente y reducir la pobreza. Los efectos de la ineficiencia en la problemática son claramente estocásticos y no están correlacionados con la variable ecológica ni la pobreza de los productores forestales en los años de observación.

Este resultado es más compatible con los datos de distribución normal analizados que con la normal-trucada, siendo esto consistente con otros estudios de fincas (Ahmad \& Bravo-Ureta, 1996).

El cuadro 4 presenta la distribución de frecuencia de la eficiencia técnica de las fincas estudiadas durante el periodo estudiado.

Se puede notar que del 30\% de las fincas estudiadas en el año 1998, la eficiencia técnica se concentra en el rango entre $11 \%$ a $20 \%$; el 20\% de las fincas 
se ubican en el rango $31 \%$ a $50 \%$; y el $15 \%$ se ubican en el rango de $1 \%$ a $10 \%$. En cambio, durante el año 2001, el $25 \%$ se concentra entre $21 \%$ a $30 \%$ y el $20 \%$ se ubica entre $31 \%$ a $50 \%$. Sin embargo, durante el año 2005 , el $85 \%$ se ubica entre el $1 \%$ y $10 \%$.

Cuadro 4. Frecuencias de eficiencias técnicas 1998-2005

\begin{tabular}{|l|c|c|c|}
\hline Frecuencias & 1998 & 2001 & 2005 \\
\hline $01-0.10$ & 3 & 2 & 17 \\
\hline $0.11-0.20$ & 6 & 2 & 1 \\
\hline $0.21-0.30$ & 2 & 5 & \\
\hline $0.31-0.40$ & 4 & 4 & \\
\hline $0.41-0.50$ & 4 & 4 & \\
\hline $0.51-0.60$ & & 1 & 1 \\
\hline $0.61-0-70$ & & 2 & 1 \\
\hline $0.71-0.80$ & 1 & & \\
\hline $0.81-0.90$ & & & \\
\hline $0.91-1$ & & & \\
\hline Fincas & 20 & 20 & 20 \\
\hline
\end{tabular}

Los resultados del último período analizado pueden explicarse porque en ese año los productores no talaron y no incurrieron en costos de abonos orgánicos y por consiguiente no generaron ingresos. Recordemos que el destino de sus parcelas es para cultivos temporales de seguridad alimentaria (ver cuadro 6 en anexos).

\section{Conclusiones y discusión}

El objetivo de este artículo fue medir el nivel de eficiencia técnica de los productores(as) de los hogares con fincas forestales que desarrollaron actividad económica de la tala de árboles, además explicar la ineficiencia como una problemática ambiental y valorar su impacto en el desarrollo local sostenible de los hogares que despliegan este tipo de actividad (ver gráficos 1 y 2 en anexos).

El impacto de la política ambiental en materia de educación forestal y medidas para mitigar los efectos de la deforestación en la economía de los hogares rurales ha sido explicado por el impacto ecológico (abonos orgánicos) y la pobreza de los hogares de acuerdo al modelo de desarrollo local sostenible estudiado con función de producción estocástica. La eficiencia técnica de los productores(as) que aportaron jornales con alimentación, sin alimentación, y trabajadores permanentes, obtuvieron una elasticidad más representativa que la contribución a reducir el costo de la canasta básica. Esto se debe en parte a que la mayoría de los productores 
no redujeron la pobreza, impactando negativamente en el ambiente. La eficiencia técnica promedio en los años de estudio fue 24\% (eficiencia técnica/distancia de la frontera). En 1998, la eficiencia técnica en promedio geométrico fue de $28 \%$, alcanzando un máximo de 74\%, en el 2001 este promedio subió a 35\%, al igual que su máximo en $69 \%$, en el 2005 este promedio se redujo a $7 \%$ y su máximo a $63 \%$ (Ver cuadro 5 y gráficos 1 y 2 en anexos). Esta tendencia se justifica porque en 2005 solamente tres productores talaron (ver cuadro 6 en anexos).

En resumen, la eficiencia técnica en promedio geométrico experimentó una tendencia decreciente. Es decir que las acciones de las políticas ambientales orientadas a reducir la deforestación impactaron negativamente en el modelo de desarrollo local. El efecto de la ineficiencia fue explicado por el gasto y la pobreza de los hogares. Los productores(as), en sus actividades económicas no están conscientes de los beneficios de la utilización de abonos orgánicos en los procesos productivos para evitar la deforestación, de tal manera que su impacto fue negativo. La contribución al gasto de consumo anual de los hogares rurales y la pobreza deben ser consideradas por los tomadores de decisiones y actores locales en función de mitigar la problemática de la tala de árboles. Valorando estos resultados desde una perspectiva ecológica (Gómez Sal, 2004), podemos sugerir la necesidad de promover la educación ambiental (Zúniga, 2009) en los productores(as) y suscitar medidas alternativas de producción agropecuaria para mitigar el proceso de la deforestación generando ingresos para reducir la pobreza. Los objetivos apuntarían hacia mejorar la eficiencia técnica de las unidades productivas de tal forma que permita incrementar la contribución de éstas al gasto anual de los hogares rurales.

Cuadro 5. Promedios geométricos de la eficiencia técnica 1998-2005

\begin{tabular}{|l|c|c|}
\hline Año & Promedio \% & Máximo \\
\hline 1998 & 28 & 74 \\
\hline 2001 & 35 & 69 \\
\hline 2005 & 7 & 63 \\
\hline Panel Datos & 24 & 74 \\
\hline \multicolumn{2}{|l|}{ Panel datos 20 fincas por año y 60 observaciones } \\
\hline
\end{tabular}

En resumen, el modelo de desarrollo local sostenible con función de producción frontera estocástica domina el promedio de la función de producción. Los efectos de la ineficiencia explicada por el gasto y la pobreza de los hogares explican el modelo DLS y su impacto en la política ambiental con los resultados anteriormente discutidos. 


\section{Referencias bibliográficas}

Ahmad, M., Bravo-Ureta, B. (1996). Technical efficiency measures for dairy farms using panel data: a comparison of alternative model specification. J Prod Anal, (7), 399-415.

Aigner, D.J., Lovell, C.A.K. \& Schmidt, P. (1977). Formulation and Estimation of Stochastic Frontier Production Function Models. Journal of Econometrics, (6), 21-37.

Atienza Serna, L. (1992). Del proteccionismo a las nuevas tendencias del desarrollo rural. (Estrategias del futuro). Revista de Estudios Agro sociales, (162), 310-332.

B.I.R.F. (1975). Desarrollo rural: documentos de política sectorial. Washington: Banco Mundial.

Barke, M., \& Newton, M. (1995). La iniciativa comunitaria leader y el desarrollo rural en España: estudio comparativo de la Alpujarra (Almería Granada) y la Loma (Jaen). Revista de Estudios Regionales, (41), 39-64.

Battese, G.E. \& Coelli. T. J. (1995). A model for Technical Inefficiency Effects in a Stochastic Frontier Production Function for Panel Data. Empirical Economics, (20), 325-332.

Battese, G.E. \& Coelli, T. J. (1992). Frontier Production Functions. Technical Efficiency and Panel Data: With Application to Paddy Farmers in India. Journal of Productivity Analysis, (3), 153-169.

Battese, G.E. \& Coelli. T. J. (1988). Prediction of Firm-Level Technical Efficiencies with a generalized Frontier Production Function and Panel Data. Journal of Econometrics, (38), 387-399.

Baurer, P.W. (1990). Recent Development in the Econometric Estimation of Frontier. Journal of Econometrics, (46), 39-56.

Berger, G. (1992). Social Structure and rural development in the Third World. Cambrige: Cambrige University Press.

Bifani, P. (1984). Desarrollo y Medio Ambiente. Madrid: MOPU. Monografías de D.G.M

Boekena, F. (1989). Desarrollo local y mercado de trabajo. La experiencia holandesa, a través de la teoría de redes. Revista Estudios Territoriales, (1), 57-78.

Bote Gómez, V. (1990). Turismo en el espacio rural. Rehabilitación del patrimonio social cultural y de la economía rural. Madrid: Ed. Popular.

Carbonell, R. (1992). Estrategia de desarrollo rural en los pueblos Guaranies. Barcelona: Antoni Bosch.

Carletto, G. (2010). Improving the Availability, Quality and Policy-Relevance of Agricultural Data: The Living Standards Measurement Study-Integrated Surveys on Agriculture. Third Wye City Group Global Conference on Agricultural and Rural Household Statistic. Washington, D.C 24-25 May 2010.

Chombart de Lauwe, P.H. (Coord). (1988). Culture-action des groupdominés: rapport à léspaceetdevelopment local. Associatino de Recherche Coopétative Internacionales. Paris: L 'Harmattan.

Cordero Mestanza, G. (1990). La planificación Regional en España. Los planes de desarrollo y reconversión regional. Madrid: Información Comercial Española. 


\section{Marzo, 1990.}

Crosby, A. (1994). Interpretación ambiental y turístico rural. Madrid: C.E.F.A.T.

Crosby, A. (1993). El desarrollo turístico sostenible en el medio rural. Madrid: C.E.F.A.T.

Dios Palomares, R. (2002). Análisis de interpretación de los parámetros de relación de varianzas en el modelo de frontera estocástica. Estudios de Economía Aplicada, 20, (II), 365-379.

Dyson, K. (Ed.). (1998). Local authorities and new technologies: the Europeans dimension. London: Croom Helm.

Entrena Durán, F. (1995). De la reforma agraria al desarrollo rural. Revista Política y Sociedad, (20), 125-141.

FAO. (1993). El alivio de la pobreza rural: tendencias y políticas. Roma: FAO.

Faure, R. (1979). Medio local y Geografía viva. Barcelona: Ed. Laia.

Forsund, F.R., Lovell, C.A.K. \& Schmidt, P. (1980). A Survey of Frontier Production Functions and of their Relationship to Efficiency Measurement. Journal of Econometrics, (13), 5-25.

Fortun, J.E. (1973). Educación y desarrollo rural. México: Instituto Indigenista Interamericano.

Gavira Álvarez, L. (1993). Segmentación del mercado de trabajo rural y desarrollo: el caso de Andalucía. Madrid: M.A.P.A.

Gómez Sal, A. (1998). Valoración multicriterio del desarrollo a escala local. Ecosistemas, (24/25), 40-48.

Gómez Sal, A. (2001). Aspectos ecológicos de los sistemas agrícolas. Las dimensiones del

desarrollo. En J. Labrador \& M.A. Itieri (Eds.). Agroecología y Desarrollo. Madrid: Mundi-Prensa.

Gómez Sal, A. (2004). Sostenibilidad ecológica: espacios y oportunidades para un reto inaplazable. Quórum, (10), 23-43.

Greene, W.H. (1993). The Econometric Approach to Efficiency Analaysis. En H.O. Fried, C.A.K. Lovell \& S.S. Schmidt (Eds.). The Measurement of Productive Efficiency. (pp. 3-91). New York: Oxford University Press.

Hoggart, K. (1987). Rural development: a geographical perspective. London: CroomHelm. Huang, C.J. \& Liu, J-T. (1994). Estimation of a non-neutral stochastic frontier production function. Journal of Productivity Analysis, (5),171-180

Instituto Nacional de Información y Desarrollo [INIDE]. (1998-2005). Base de datos de las Encuestas de Medición de Nivel de Vida, EMNV. Recuperado el 20 de mayo de 2010 de www. Inide. gob.ni

Kayser, B. (1990). La renaissance rurale: sociologie des capmagnes du monde occidental. Paris: Armand Colin.

Kumbhakar, S.C., Ghosh, S. \& McGuckin, J.T. (1991). A generalized production frontier approach for estimating determinants of inefficiency in U.S. dairy farms. J Bus Econ Stat, (9), 279-286.

Meeusen, W. \& van den Broeck, J. (1977). Efficiency Estimation from CobbDouglas Production Functions with Composed Error. International Economic Review, (18), 435-444.

Meilán Gil, J.L. (1971). El territorio protagonista de desarrollo. Madrid: C.S.C.C.I.N.

Oakley, P. (1993). Proyectos con la población: la práctica de la participación en el desarrollo 
rural. Madrid: M.T.S.S.

Potter, R.B. \& Unwng, T. (1989). The Geogrphy of urban-rural interaction in developing countries. London: Routledge.

Reifschneider, D. \& Stevenson, R. (1991). Systematic Departures from the Frontier: A Framework for the Analysis of Firm Inefficiency. International Economic Review, (32), 715-723.

Rodríguez Fraguas, J.A. (1993). El desarrollo rural, alternativa real...o cortina de humo. Revista Distribución y Consumo, 3 (8), 34-41.

Ruíz Pérez, M. (1993). Infraestructuras y desarrollo rural. Revista El Campo, (127), 279-284.

Sambergs, A.E. (1979). Nuevo empleo rural: una visión y sus posibilidades. Agricultura y Sociedad (Octubre/Diciembre) no 13, Madrid. M.A.P.A.

Schmidt, P. (1986). Frontier Production Functions. Econometric Reviews, (4),289-328.

Stevenson. R.E. (1980). Likelihood Functions for Generalized Stochastic Frontier Estimation Journal of Econometrics, (13), 57-66.

Urbina, R. (2005). Inventario de politicas agroambientales en Nicaragua. Managua: Instituto Interamericano de Cooperación para la Agricultura (IICA).

Valcárcel-Resalt, G. (1992). Balance y perspectivas del desarrollo local en España. En C. del Canto (Ed.) Desarrollo Rural. Ejemplos Europeos. Madrid: IRYDA, Ministerio de Agricultura y Pesca.

Whatmore, S., Marsder, T. \& Lowe, P. (1993). Cambio tecnológico y medio ambiente rural. Madrid: M.A.P.A (S.G.T).

Zapata Martelo, E. (1994). Mujeres rurales ante el nuevo milenio: desde la teoría del desarrollo rural hacia la concepción del género en el desarrollo. México: Colegio de Posgraduados.

Zúniga, C. A. (2009). Análisis del indice de MALMQUIST DEA con un output orientado aplicado a la actividad forestal en NICARAGUA 1998-2005. IV Simposio Agroforestal "Contribución de los sistemas agroforestales de manejo sostenible de la tierra para mitigación y adaptación al cambio climático", Universidad Nacional Autónoma de Nicaragua, León, Nicaragua, 5-6 de noviembre de 2009. Recuperado el 10 de mayo de 2010 de http://purl.umn.edu/56198

Zúniga, C. A. (2010a). Nuevo Modelo de Participación Ciudadana: Identificación de Sistemas de Producción agropecuarios y Forestales en los Departamentos de León y Chinandega, 2008-2009. Recuperado el 10 de mayo de 2010 de http:// purl.umn.edu/56692

Zúniga, C. A. (2010b). Comparisons of LSMS-ISA data collection and dissemination efforts in Central America. The Wye Group Global Conference on Agricultural and Rural Household Statistic; Economic Research Service, U.S Department of Agricultural Washington, D.C., 24-25 de mayo de 2010. Recuperado el 10 de julio de 2010 de http://purl.umn.edu/90751http://typo3.fao.org/ fileadmin/templates/ess/pages/rural/wye_city_group/2010/3rd_Wy e_Conference.html 


\section{Anexos}

Cuadro 6. Panel de datos

\begin{tabular}{|l|c|c|c|c|c|c|c|}
\hline No formulario & firm & year & Output & Labour & Contrib & organic & Poverty \\
\hline 43900 & 1 & 1 & 6 & 6 & 3 & 0 & 1 \\
\hline 44800 & 2 & 1 & 7 & 7 & 4 & 163 & 0 \\
\hline 55300 & 3 & 1 & 5 & 5 & 4 & 0 & 0 \\
\hline 55400 & 4 & 1 & 6 & 7 & 3 & 0 & 0 \\
\hline 83800 & 5 & 1 & 10 & 5 & 2 & 0 & 0 \\
\hline 105700 & 6 & 1 & 7 & 7 & 4 & 0 & 0 \\
\hline 126600 & 7 & 1 & 6 & 3 & 4 & 0 & 0 \\
\hline 131700 & 8 & 1 & 7 & 7 & 3 & 0 & 1 \\
\hline 190400 & 9 & 1 & 7 & 6 & 2 & 0 & 0 \\
\hline 225000 & 10 & 1 & 6 & 8 & 3 & 1712 & 0 \\
\hline 234600 & 11 & 1 & 7 & 8 & 3 & 0 & 0 \\
\hline 236900 & 12 & 1 & 6 & 7 & 3 & 0 & 0 \\
\hline 258400 & 13 & 1 & 7 & 6 & 3 & 0 & 0 \\
\hline 301000 & 14 & 1 & 5 & 2 & 3 & 0 & 1 \\
\hline 343500 & 15 & 1 & 8 & 9 & 3 & 0 & 0 \\
\hline 362100 & 16 & 1 & 6 & 8 & 2 & 0 & 0 \\
\hline 398500 & 17 & 1 & 7 & 11 & 3 & 815 & 1 \\
\hline 398700 & 18 & 1 & 7 & 8 & 4 & 0 & 0 \\
\hline 430500 & 19 & 1 & 5 & 8 & 5 & 0 & 0 \\
\hline 430900 & 20 & 1 & 5 & 8 & 3 & 408 & 1 \\
\hline 439 & 1 & 2 & 6 & 0 & 3 & 0 & 1 \\
\hline 448 & 2 & 2 & 6 & 6 & 4 & 0 & 1 \\
\hline 553 & 3 & 2 & 7 & 0 & 4 & 0 & 0 \\
\hline 554 & 4 & 2 & 6 & 0 & 3 & 0 & 0 \\
\hline 838 & 5 & 2 & 8 & 0 & 1 & 0 & 0 \\
\hline 1057 & 6 & 2 & 7 & 10 & 3 & 0 & 0 \\
\hline 1266 & 7 & 2 & 6 & 0 & 4 & 114 & 1 \\
\hline 1317 & 8 & 2 & 5 & 8 & 3 & 0 & 1 \\
\hline 1904 & 9 & 2 & 6 & 0 & 2 & 0 & 1 \\
\hline 2250 & 10 & 2 & 7 & 7 & 3 & 0 & 0 \\
\hline 2346 & 11 & 2 & 6 & 0 & 3 & 0 & 0 \\
\hline 2369 & 12 & 2 & 3 & 0 & 3 & 610 & 0 \\
\hline 2584 & 13 & 2 & 6 & 0 & 2 & 0 & 0 \\
\hline & & & & & & & \\
\hline
\end{tabular}




\begin{tabular}{|c|c|c|c|c|c|c|c|}
\hline 3010 & 14 & 2 & 7 & 0 & 2 & 0 & 1 \\
\hline 3435 & 15 & 2 & 7 & 8 & 3 & 0 & 1 \\
\hline 3621 & 16 & 2 & 9 & 0 & 2 & 0 & 1 \\
\hline 3985 & 17 & 2 & 8 & 0 & 3 & 858 & 0 \\
\hline 3987 & 18 & 2 & 7 & 9 & 3 & 0 & 0 \\
\hline 4305 & 19 & 2 & 7 & 10 & 5 & 0 & 0 \\
\hline 4309 & 20 & 2 & 8 & 11 & 3 & 0 & 0 \\
\hline 439 & 1 & 3 & 8 & 6 & 3 & 0 & 1 \\
\hline 448 & 2 & 3 & 5 & 0 & 4 & 0 & 1 \\
\hline 553 & 3 & 3 & 0 & 0 & 4 & 0 & 1 \\
\hline 554 & 4 & 3 & 0 & 0 & 3 & 0 & 1 \\
\hline 838 & 5 & 3 & 0 & 0 & 2 & 0 & 0 \\
\hline 1057 & 6 & 3 & 0 & 11 & 4 & 0 & 1 \\
\hline 1266 & 7 & 3 & 0 & 9 & 4 & 0 & 1 \\
\hline 1317 & 8 & 3 & 0 & 12 & 3 & 0 & 1 \\
\hline 1904 & 9 & 3 & 0 & 0 & 2 & 0 & 0 \\
\hline 2250 & 10 & 3 & 0 & 0 & 3 & 0 & 1 \\
\hline 2346 & 11 & 3 & 0 & 0 & 3 & 0 & 0 \\
\hline 2369 & 12 & 3 & 0 & 0 & 3 & 0 & 1 \\
\hline 2584 & 13 & 3 & 0 & 0 & 3 & 0 & 0 \\
\hline 3010 & 14 & 3 & 0 & 0 & 3 & 0 & 0 \\
\hline 3435 & 15 & 3 & 0 & 0 & 3 & 0 & 1 \\
\hline 3621 & 16 & 3 & 0 & 0 & 2 & 0 & 0 \\
\hline 3985 & 17 & 3 & 0 & 11 & 3 & 0 & 1 \\
\hline 3987 & 18 & 3 & 0 & 7 & 4 & 0 & 1 \\
\hline 4305 & 19 & 3 & 8 & 11 & 5 & 0 & 1 \\
\hline 4309 & 20 & 3 & 0 & 0 & 3 & 0 & 0 \\
\hline
\end{tabular}

Fuente: EMNV 98-01-05 Datos deflactados y logaritmo natural aplicado. 
Gráfico 1: Eficiencia Técnica de las fincas forestales Panel de datos 1998-2005
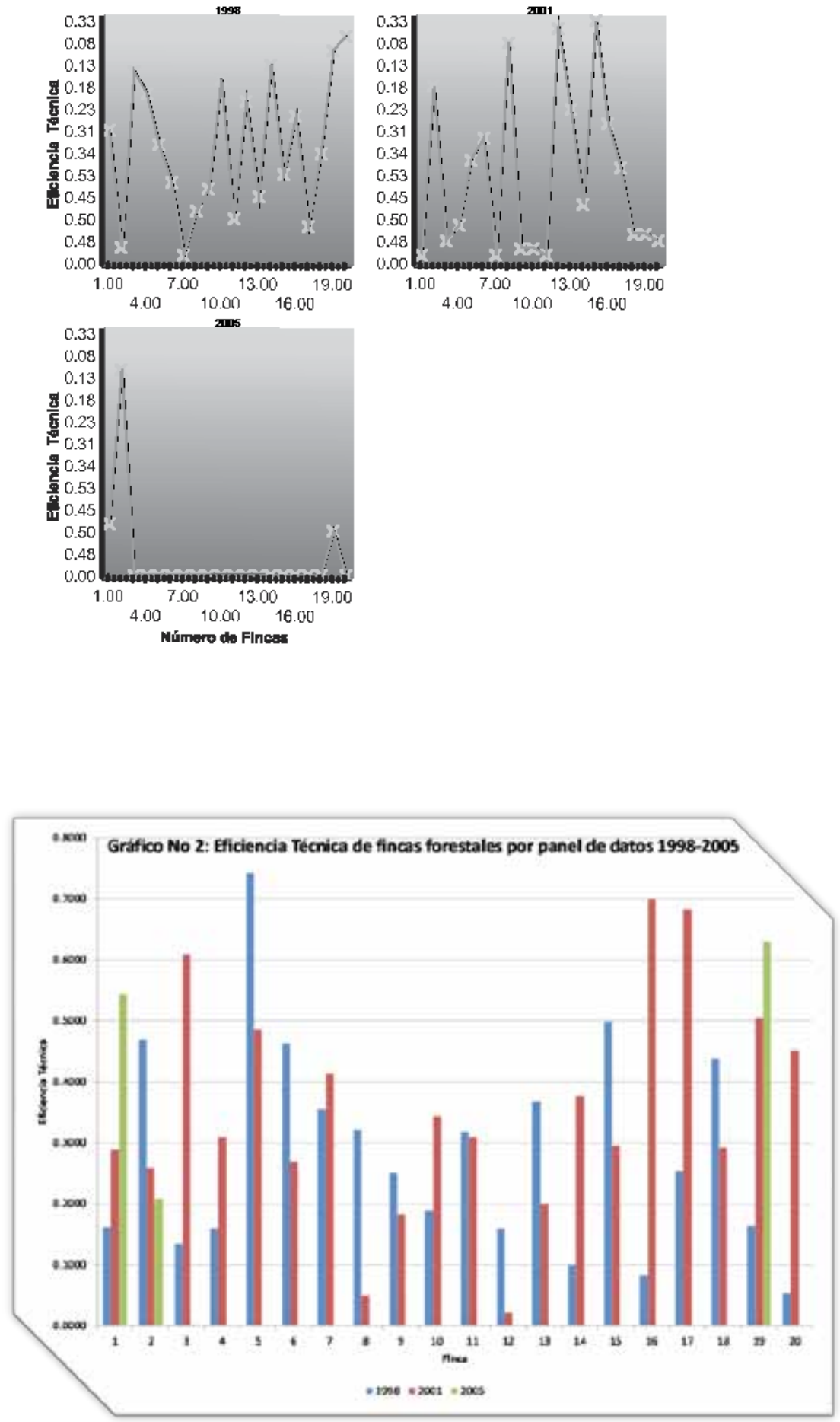